\title{
Diseño e implementación del Repositorio de Información del Centro de Estudios CAD/CAM en la Universidad de Holguín: tecnología para la gestión de la producción científica
}

\author{
Nelvis Reyes Fernández ; Rosa Margarita Rodríguez Fernández²; Luis Orlando Aguilera \\ García $^{3}$; Carmen Almaguer Riverón ${ }^{4}$; Adelaida Lahera Macías ${ }^{5}$
}

Recibido: 12 de julio 2017 / Aceptado: 15 de noviembre de 2017

Resumen. Se presenta el diseño e implementación del Repositorio de Información del Centro de Estudios CAD/CAM, el que junto a la biblioteca universitaria de la sede "Oscar Lucero Moya de la Universidad de Holguín constituyó el contexto de la investigación. Fue desarrollada una primera etapa que tuvo la finalidad de diagnosticar el conocimiento que sobre los RI presentaban, tanto los trabajadores del centro de estudios como los de la biblioteca universitaria. Para realizar la colecta de la información se aplicó como técnica cuantitativa el cuestionario (con preguntas cerradas y abiertas) a los profesores del centro de estudio y entrevistas del tipo estructurada a los bibliotecarios. Como técnica cualitativa se desarrolló la observación participante que posibilitó obtener información sobre el comportamiento de los conocimientos de los bibliotecarios con respecto al trabajo en la plataforma Dspace tanto antes como después de las acciones de capacitación. Con los resultados fue posible planificar y desarrollar un programa de formación para los trabajadores de la biblioteca y se obtuvieron elementos que fueron considerados para desarrollar la segunda etapa de estudio en la que se diseñó e implementó el repositorio. En esta etapa se utilizó como técnica cualitativa el grupo focal con los investigadores del centro de estudio y además una entrevista estructurada. Como resultado quedó defina la estructura del repositorio, el flujo documental y los roles de los actores, tanto bibliotecarios como profesores. De esta forma quedaron listos tanto la plataforma como los recursos humanos para poder desarrollar el RI que actualmente se encuentra disponible en http://repositorio.uho.edu.cu/jspui/.

Palabras clave: Repositorio Institucional; Biblioteca universitaria; Centro de estudios CAD/CAM; Universidad de Holguín, Gestión de información

1 Departamento de Ciencias de la Información. Universidad de Holguín, Cuba.

E-mail: nreyes@uho.edu.cu

2 Departamento de Ciencias de la Información. Universidad de Holguín, Cuba.

E-mail: rmargarita@uho.edu.cu

3 Departamento de Filosofía. Universidad de Holguín, Cuba.

E-mail: loag@uho.edu.cu

4 Departamento de Filosofía. Universidad de Holguín, Cuba.

E-mail: calmaguer@uho.edu.cu

5 Biblioteca "Benito Juárez". Universidad de Holguín, Cuba.

E-mail: saicam@uho.edu.cu 


\title{
[en] Design and implementation of the Information Repository of the Study Center CAD/CAM at the University of Holguin: technology for the management of scientific production
}

\begin{abstract}
The design and implementation of the Information Repository of the CAD/CAM Study Center is presented the one that next to the university library of the campus "Oscar Lucero Moya of the University of Holguín constituted the context of the investigation. It was developed a first stage that had the purpose of diagnosing the knowledge that about the RI presented, both the workers of the study center and those of the university library. To collect the information, the questionnaire (with closed and open questions) was applied as a quantitative technique to the teachers of the study center and structured interviews to the librarians. As a qualitative technique, participant observation was developed, which made it possible to obtain information about the behavior of librarians' knowledge regarding work on the DSpace platform both before and after the training actions. With the results it was possible to plan and develop a training program for library workers and elements were obtained that were considered to develop the second stage of study in which the repository was designed and implemented. In this stage, the focus group was used as a qualitative technique with the researchers of the study center and also a structured interview. As a result it was defined the structure of the repository, the documentary flow and the roles of the actors, both librarians and teachers. In this way both the platform and human resources were ready to develop the RI that is currently available in http://repositorio.uho.edu.cu/jspui/.

Keywords: Institutional Repository; University Library; CAD/CAM Studies Center; University of Holguin; Information management.
\end{abstract}

Sumario. 1. Introducción. 2. Desarrollo. 3. Resultados. 4. Conclusiones. 5. Referencias bibliográficas

Cómo citar: Reyes Fernández, N., Rodríguez Fernández, R.M., Aguilera García, L.O., Almaguer Riverón, C., Lahera Macías, A. (2017) Diseño e implementación del Repositorio de Información del Centro de Estudios CAD/CAM en la Universidad de Holguín: tecnología para la gestión de la producción científica, en Revista General de Información y Documentación 27 (2), 317-340.

\section{Introducción}

En Cuba se han realizado numerosos esfuerzos por conectar el conocimiento, la ciencia y la tecnología a las demandas sociales, con éxito variable, según los casos (Núñez - Jover y Castro-Sánchez 2005: 2 y 9). Las universidades juegan en ese contexto un rol esencial y tienen a su favor el poderoso recurso de la educación continua para formar y renovar las habilidades inherentes al comportamiento innovativo. Por eso las actividades de capacitación, pregrado y postgrado, se convierten en vehículos importantes de las actividades de ciencia e innovación.

La actividad científica genera en las universidades grandes cantidades de conocimientos que se explicitan, fundamentalmente, en documentos como: trabajos de diplomas (pregrado), tesis doctorales, de maestría y especialidades, informes y artículos científicos. Esos conocimientos, considerando su naturaleza de bien público, deben retornar a la sociedad que los sustenta como respuesta a las demandas sociales, no obstante aún una parte de ellos se estancan, están dispersos o se pierden y una de las causas es que no son debidamente almacenados, categorizados y visualizados de forma tal que se facilite su acceso y apropiación social.

Para resolver la necesidad del acceso directo a los contenidos de la producción científica de las organizaciones han aparecido los repositorios institucionales; los 
mismos "son creados dentro de las instituciones para visualizar y preservar su propia producción intelectual. En el ambiente universitario los repositorios institucionales son colecciones que capturan y preservan la producción intelectual de una o más universidades, son un determinado conjunto de servicios que una universidad ofrece a los miembros de su comunidad a fin de gerenciar y diseminar materiales digitales creados por la institución y por miembros de la comunidad" (Lynch 2003: 2).

Según Castaño Muñoz y Restrepo (2016: 60) "los repositorios institucionales vienen a ofrecer la posibilidad de almacenar diferentes tipos de contenidos digitales, como artículos, libros, monografías en texto completo que se encuentren en varios formatos, como PDF, Word, Power Point y también contenidos multimedia, como videos, audios, fotografías, entre otros"

Estos autores consideran que dentro de las ventajas de esta herramienta está la indexación del contenido completo, de tal manera cuando el usuario realiza una búsqueda libre lo hará no solo en los campos definidos por los analistas y catalogadores, sino en el texto completo y se visualizará dentro de los resultados, por otra parte los repositorios se indexan e integran a Internet por lo que sus contenidos pueden ser recuperados por los buscadores.

Los Repositorios Institucionales (en adelante RI) soportados en infraestructuras tecnológicas engloban en su desarrollo además a las personas. Los gestores de información que administran el RI desde la biblioteca universitaria o cualquier otra entidad y los investigadores configurados en un doble papel de productores y consumidores de información transitarán por un proceso paulatino y constante de empoderamiento en su papel de protagonistas del desarrollo científico tecnológico e innovativo dentro de la organización. Mientras, por las características de los RI, también se facilita la conexión entre investigadores por lo que se potencian los proyectos colaborativos y las redes de conocimiento.

De esta forma el RI se analiza desde la perspectiva de la construcción social de una tecnología, considerando que "los procesos de producción y de construcción social de la utilidad y el funcionamiento de las tecnologías constituyen dos caras de una misma moneda de la adecuación socio-técnica: la utilidad de un artefacto o conocimiento tecnológico no es una instancia que se encuentra al final de una cadena de prácticas sociales diferenciadas, sino que está presente tanto en el diseño de un artefacto como en los procesos de resignificación de las tecnologías en los que participan diferentes grupos sociales relevantes (Thomas, 2009: 19). En este caso los grupos sociales relevantes: investigadores, gestores de información y comunidad universitaria y extrauniversitaria entran en interacción con la tecnología y se apropian de ella como vía para el acceso a la información y el incremento del conocimiento.

El enfoque en la construcción social de una tecnología permite estudiar la interrelación y visión de los grupos sociales sobre la misma con una visión holística del fenómeno. Siguiendo a Bijker (1995) se considera que los sentidos que los grupos sociales relevantes atribuyen a la tecnología determinan su éxito o fracaso. De esta forma puede ser aceptada, rechazada o criticada, de ahí la necesidad de estudiar la percepción y las significaciones construidas por los actores sobre ella, así como su preparación para apropiarse de ella. 
Según Crow (2002: 20) todos los involucrados en el proceso de desarrollo de un RI se verán afectados por los cambios que éste produce en la estructura de las comunicaciones académicas, dentro de ellos los bibliotecarios, estudiantes, investigadores y las editoriales. De esta forma es necesario estimular la comprensión de los objetivos y la política por parte de los grupos sociales relevantes que intervienen en la implementación de un RI pues ellos pueden desempeñar un papel importante si se trabaja desde una visión participativa y de cooperación.

Para estabilizar los significados atribuidos al RI por parte de los grupos sociales relevantes y lograr su participación se debe partir de un diagnóstico de dichos significados; esto permitirá desarrollar acciones de aprendizaje para que esos actores conozcan al detalle y con la debida transparencia todos los aspectos que median en el enfoque de acceso abierto a la información y la tecnología que lo sustenta, así como el rol que ellos juegan en este sistema. Debe definirse además una política que estimule la publicación de los resultados científicos y considere los elementos de la gestión documental, de la información y del conocimiento.

$\mathrm{Al}$ analizar los RI desde la perspectiva de los grupos sociales relevantes, Rieger (2008) establece que esos grupos son los siguientes:

1. Instituciones de investigación: Dentro de ellas las bibliotecas que despliegan un importante papel relacionado con la instalación y mantenimiento del RI y la gestión de programas de entrenamiento dentro de la organización.

2. Informáticos, desarrolladores y bibliotecarios.

3. Usuarios finales: Dentro de ellos investigadores, y estudiantes avanzados que publican resultados de sus investigaciones, son consumidores de la información y a la vez contribuyen mediante el autoarchivo de su producción científica al desarrollo del RI.

4. Comunidades de acceso abierto.

5. Editoriales: Incluye sociedades científicas y académicas, autores individuales y las editoriales académicas, entre otros.

Hernández Pérez, Rodríguez Mateos y Bueno de la Fuente (2007: 199) consideran que más allá de las soluciones tecnológicas por las que se opte en la conformación de un RI, las tareas que debe emprender la biblioteca son las siguientes:

1. Definición de las políticas del repositorio.

2. Planificación y gestión del proyecto de repositorio, donde es fundamental el análisis de los costos.

3. Elección del paquete de software en el que implementar el repositorio.

4. Definición de las estrategias para el arranque del repositorio.

5. Definición y puesta en marcha de las estrategias de publicidad del repositorio.

6. Seguimiento, evaluación y mejora de los servicios implementados.

Por otra parte la decisión de asumir la conformación del RI y con ello el acceso abierto a la información puede ser asumida de diferentes formas que son conocidas 
como mandatos. La Confederación de Repositorios de Acceso Abierto COAR (2013: 8) apunta que los mandatos pueden ser implementados de diversas maneras, dependiendo del contexto institucional, pero generalmente son registrados en una de dos formas: a través de mandatos institucionales de "arriba hacia abajo", que son iniciados por la administración de una institución o un administrador de alto mando; o de "abajo hacia arriba", con políticas desarrolladas, votadas y aprobadas por los miembros de la institución que son parte de los consejos de administración de las universidades, como el claustro universitario. Existen también las políticas voluntarias; sin embargo en este caso son muy bajos los índices de autoarchivo de los contenidos por parte de los investigadores.

En Europa los mandatos institucionales de "arriba hacia abajo" son los más comunes. Ejemplos destacados son el de la Universidad de Liège (Bélgica) y el de la Universidad de Minho (Portugal). Ambas instituciones han tenido mandatos establecidos por varios años y sus programas de Acceso Abierto, que implican promoción y servicios de repositorios, se encuentran fuertemente vinculados a estos mandatos. En contraste, en América del Norte las políticas han sido implementadas más desde un enfoque de "abajo hacia arriba" en el Instituto de Tecnología de Massachusetts (MIT), la Universidad de Educación de Stanford y la Universidad de Duke, todas de Estados Unidos de Norteamérica, la política se debe acompañar de una estrategia de promoción, capacitación y apoyo a los investigadores (COAR 2013).

Como ya se ha tratado, el rol de las bibliotecas en la conformación de los RI es fundamental, por lo que los recursos humanos de esta institución verán cambiar un tanto sus roles. En el decreto-ley no. 271 "De las bibliotecas de la República de Cuba" se define la Biblioteca Universitaria como "un centro para el aprendizaje, la docencia, la investigación y las actividades relacionadas con el funcionamiento y la gestión de las universidades, constituidos por los fondos bibliográficos, documentales, audiovisuales y digitales adquiridos por los diversos departamentos, centros y servicios, cualquiera que sea la forma de su adquisición, incluidos los legados, y aquellos donados a favor de la universidad por otras instituciones"(Ministerio de Justicia de la República de Cuba, 2010: 874).

Para Babini González, López Medina y Medice (2010: 76) "los RI son una gran oportunidad de mejorar los servicios tradicionales en las bibliotecas universitarias. Suman así valor agregado, gracias a la incorporación del acceso al texto completo de los documentos descritos en las bases tradicionales de datos bibliográficos y en los catálogos colectivos ya existentes. Las bibliotecas tienen también experiencia en la gestión de colecciones, en la automatización de sus procesos y en la descripción de documentos. Desde ese lugar, han sido las principales promotoras para la creación de colecciones digitales con la producción de su institución"

A pesar de los beneficios que reportan los RI, en Cuba no se constata la existencia de una política que gobierne y normalice el diseño e implementación de RI en la red de bibliotecas del Ministerio de Educación Superior, aunque se han dado pasos, aún es incipiente el desarrollo de los repositorios. Al respecto, Álvarez Fernández, (2016: 3) indica que "según el Registro Mundial de Repositorios de 
Acceso Abierto (Registry of Open Access Repositories-ROAR), Cuba solo contaba en ese momento con seis RI de Acceso Abierto.

En el afán por insertarse en la aplicación de esta tecnología, en el año 2013, seis universidades cubanas se unieron a universidades belgas en la denominada "Red de cooperación universitaria para el fortalecimiento del rol de las TIC en las universidades cubanas para el desarrollo de la sociedad" El proyecto 3 de esta red se enfoca al "Soporte de las TIC para los procesos educativos y la gestión del conocimiento en la Educación Superior" (ELINF) lo que permitió dar pasos para la creación de los RI Institucionales, a partir de una política cooperada.

Las bibliotecas que forman parte de este proyecto son las siguientes: Universidad "Hermanos Sainz" de Pinar del Rio, Universidad de Las Ciencias Informáticas (UCI), Universidad Central "Marta Abreu" de Las Villas, Universidad "Ignacio Agramonte" de Camagüey y la Universidad de Holguín.

Las Bibliotecas Universitarias o Centros de Documentación e Información Científico Técnica, según sea el caso en cada una de las universidades, se nombraron responsables de coordinar todo el funcionamiento y desarrollo del RI en cada centro, para ello fueron convocadas a crear la política para el desarrollo de la red de RI del Ministerio de Educación Superior. Dicha política establece los preceptos fundamentales que rigen el funcionamiento de los repositorios dentro de las universidades cubanas participantes en el proyecto antes mencionado y declara los principios de trabajo, las implicaciones de las distintas áreas y el personal vinculado directa e indirectamente con su desarrollo.

En este trabajo se asume que los RI constituyen herramientas tecnológicas para una nueva forma de comunicación de la ciencia donde se eliminan barreras económicas y de permisos relacionadas con el acceso a la información, y se reconoce que asociada a esa tecnología se manifiesta la demanda de saberes, habilidades y actitudes necesarias para interactuar con ella hasta que los actores las incorporen a sus prácticas investigativas cotidianas y la conviertan en instrumento para la innovación y el desarrollo. Por ello se consideró que para poder implementar la política era básico realizar un diagnóstico de los vacíos de conocimientos y en correspondencia diseñar las acciones de formación a desarrollar para propiciar que las personas asimilaran la tecnología desde el conocimiento, no como una imposición.

Se reconoce que aunque la política fue aprobada por mandato "de arriba hacia abajo", ésta debe acompañarse de un enfoque participativo donde la comunicación y la formación desempeñan un papel preponderante y abarque desde la alta dirección de la universidad hasta las áreas donde se desempeñan los docentes y trabajadores involucrados. Por otra parte fue necesario definir el flujo documental y de información y el rol de los actores: directivos, investigadores y la biblioteca universitaria como centro coordinador.

Luego la investigación se orientó al diseño e implementación del RI del Centro de Estudios CAD/CAM (Computer Aided Design/Computer Aided Manufacturing) a partir de la contextualización de la política de la Red de Repositorios Digitales de Información (RRDI) de universidades cubanas lo que permitió estudiar la interrelación y visión de los grupos sociales sobre la misma con una visión holística 
del fenómeno que incluyó las significaciones construidas por los actores relacionados con este proceso. De ahí que cada fase se concibió de forma participativa.

\subsection{Caracterización del Centro de Estudios CAD/CAM de la Universidad de Holguín}

Este centro de estudios fue fundado el primero de octubre de 1989 con la misión de "impartir docencia de pregrado y postgrado de alta calidad, aplicar los resultados de las investigaciones en la solución de problemas científico-técnicos dentro y fuera del país, basado en una fuerte relación universidad - entorno que ha posibilitado contribuir económicamente al sostenimiento de la universidad teniendo como base un colectivo de excelencia con una sólida formación científica en las tecnologías CAD/CAM/CAE (Computer Aided Engineering). En sus inicios enfocó sus actividades investigativas al perfeccionamiento de la Combinada Cañera Cubana y a la fabricación de implementos agrícolas.

Ha logrado importantes resultados en el diseño automatizado de herramientas de corte, troqueles, estampas y dispositivos de maquinado así como en la asimilación de las tecnologías CAD/CAM por las empresas del territorio, entre ellas las empresas de Recursos Hidráulicos y las plantas productoras de Níquel. El centro de estudios cuenta además con un programa de maestría en "Diseño y Manufactura Asistida por Computadoras", con cinco ediciones nacionales y dos en el extranjero. Actualmente está catalogado como Programa de Excelencia por la Junta de Acreditación Nacional.

En el curso académico 2014-2015 este centro contaba con una plantilla de nueve profesores investigadores, de ellos cinco doctores en ciencias técnicas (56.6\%); tres máster y un ingeniero mecánico; del total cinco profesores poseen categoría docente principal (56.6\%). Predominan los títulos académicos de doctores y máster y los profesores con categoría principal, como se muestra en el gráfico 1.

Gráfico 1. Comportamiento de la producción científica del centro de estudios CAD/CAM por categorías académica y científica. Informe del Centro de Estudios CAD/CAM 20102014.

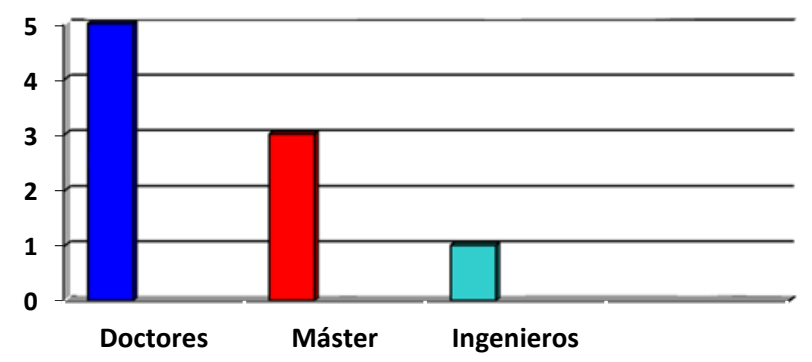


Las líneas de investigación que centran su actividad científica son las siguientes:

1. Computer Aided Design (Diseño herramental, Diseño de redes)

2. Computer Aided Process Planning (Planificación y optimización de procesos de fabricación, Sistemas CAPP en software libre y propietario)

3. Computer Aided Manufacturing (Generación de tecnologías de fabricación, Simulación de procesos de fabricación, Optimización de secuencias de manufactura)

4. Computer Aided Engineering (Análisis de Ingeniería con métodos numéricos, biomecánica computacional)

Como resultado del desarrollo de los proyectos de ciencia e innovación y de los programas de posgrado es apreciable la producción científica de este centro. En el gráfico 2 se ilustra el comportamiento de la producción científica que ha sido publicada entre los años 2010-2014 por sus investigadores. Es significativa la cantidad de publicaciones en el grupo I con 21 artículos, mientras se publican 13 en el grupo II y 3 en el grupo 3 para un total de 38 publicaciones. También han publicado libros y capítulos de libros y le han sido concedidas diferentes patentes.

Gráfico 2 Comportamiento de la producción científica del centro de estudios CAD/CAM por grupos de publicaciones. Informe del Centro de Estudios CAD/CAM 2010-2014.

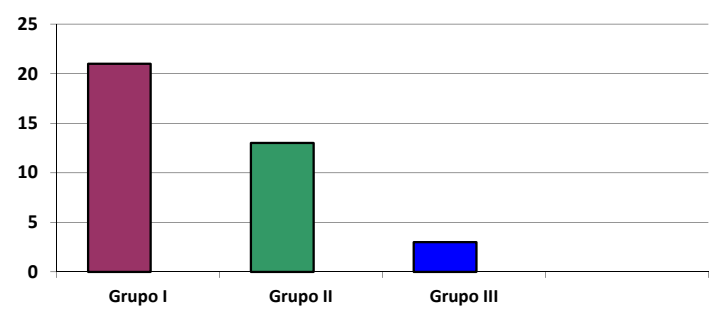

En los últimos años la labor del centro de estudios se ha enfocado en la colaboración en la solución de problemas en empresas de alto impacto en la economía y la sociedad cubana como: El Centro de Proyectos del Níquel (CEPRONIQUEL), Instituto Nacional de Recursos Hidráulicos (INRH) y vale resaltar, por su impacto social, las ayudas técnicas para discapacitados que posibilitaron las mejoras en el diseño y el proceso de producción de sillas de ruedas para la industria local cubana. Como resultado de su labor científica y la introducción de los resultados en áreas claves de desarrollo los investigadores del centro de estudios han obtenido diferentes premios, tanto a nivel individual como colectivo. Por otra parte mantienen vínculos con universidades de España, Argentina, Venezuela y Perú.

Por lo anteriormente expuesto este centro de estudios puede considerarse paradigma de la actividad de ciencia e innovación tecnológica y de la vinculación universidad-sociedad. Vale entonces apropiarse de las posibilidades que ofrecen los RI para categorizar, preservar y visualizar su producción científica que se 
revela como un importante patrimonio académico y un significativo nicho de conocimientos a movilizar, aplicar y generalizar en otros contextos nacionales e internacionales para hacer cada vez más pertinente la universidad.

En este entramado de actores sociales, aplicación de resultados y sociedad, el RI se configura como una tecnología que conecta los conocimientos que se producen en la universidad con la comunidad interna y externa donde es factible su aplicación, es de esta manera, una importante herramienta para la gestión de la información y el conocimiento universitario. Por otra parte la conformación del RI del Centro de Estudios CAD/CAM se alinea con la proyección estratégica de la universidad y por tanto del propio centro de estudios.

\subsection{Métodos y materiales}

Para alcanzar los objetivos propuestos se desarrolló un estudio descriptivo con un enfoque mixto pues se utilizaron tanto técnicas cuantitativas como cualitativas. Se manejaron de forma particular los métodos empíricos de investigación.

El contexto de la investigación lo constituyó el Centro de estudio CAD/CAM y la biblioteca universitaria "Benito Juárez de la sede universitaria "Oscar Lucero Moya de la Universidad de Holguín. A partir de los contactos realizados con el director del centro de estudio se tuvo pleno acceso a dicho lugar; en el caso de la biblioteca tres de las investigadoras forman parte de este colectivo laboral lo que facilitó la aplicación de los instrumentos de investigación.

En su primera etapa el estudio tuvo la finalidad de diagnosticar el conocimiento que sobre los RI presentaban, tanto los trabajadores del centro de estudios como los de la biblioteca universitaria. Para realizar la colecta de la información necesaria se aplicó como técnicas cuantitativa el cuestionario (con preguntas cerradas y abiertas) a los profesores del centro de estudio y las entrevistas del tipo estructurada a los bibliotecarios. Como técnica cualitativa se desarrolló la observación participante que posibilitó obtener información sobre el comportamiento de los conocimientos de los bibliotecarios con respecto al trabajo en la plataforma Dspace tanto antes como después de las acciones de capacitación. También se utilizó el método estadístico para el procesamiento de los datos obtenidos del cuestionario y las entrevistas.

En el centro de estudio se trabajó con una muestra de ocho profesores, el total de los que se encontraban laborando en Cuba al momento de la investigación. Para poder obtener y evaluar la información se aplicó un cuestionario que contenía las siguientes variables a investigar:

a) Forma de almacenamiento y preservación de las colecciones de documentos del centro de estudios en el momento en que se desarrolló la investigación.

b) Visibilidad de los resultados de las investigaciones del centro de estudio.

c) Accesibilidad a los resultados de las investigaciones.

d) Contextos de visibilidad de los resultados.

e) Conocimiento acerca de los RI. 
f) Conocimiento acerca de la ruta verde y dorada de acceso a la información como forma de comunicar los resultados científicos.

Mientras que, para poder obtener y evaluar la información sobre los trabajadores de la biblioteca, se seleccionó una muestra de 15 personas, de forma intencionada, porque son las implicadas en el trabajo en el RI, tanto el de este centro de estudio como de otras áreas de la universidad. A estas personas se le aplicó una entrevista estructurada donde se estudiaron las siguientes variables:

a) Conocimiento acerca de los RI.

b) Documentos que deben estar en el repositorio.

c) Conocimiento acerca de la ley de derecho de autor.

d) Conocimientos acerca de las políticas de los RI.

e) Conocimiento acerca de los roles de los trabajadores de la biblioteca.

f) Dudas o temores al enfrentar la tarea del trabajo en el repositorio.

g) Necesidad de capacitación.

En una segunda etapa del estudio se buscó la información que permitió obtener elementos para diseñar e implementar el repositorio, tomando en consideración las apreciaciones de los principales actores que intervienen en su gestión.

Como técnica cualitativa se utilizó el grupo focal con los investigadores del centro de estudio. A partir de los resultados obtenidos mediante el cuestionario, de los contactos sistemáticos con los investigadores del centro de estudios y con su director, se coordinó un grupo focal con el objetivo de exponer las características generales del proyecto de creación del repositorio, ganar la confianza de los investigadores al mostrarle las ventajas del repositorio como herramienta para la comunicación científica, la visualización, socialización y reutilización de los conocimientos en el marco de la ruta verde de acceso a la información, considerando que esta es una forma de sumarlos al uso del mismo. Asimismo sirvió para triangular la información obtenida con otras técnicas.

Posteriormente se aplicó una entrevista estructurada a los investigadores del centro de estudio en la que se consideraron las variables siguientes:

a) Documentos que deben estar en el repositorio.

b) Personas autorizadas a incluir trabajos en el reposito.

c) Alternativa para incluir proyectos de los estudiantes y las tesis que obtengan 3 puntos.

d) Lugar para la colección si el centro de estudios dejara de existir.

e) Alternativa a seguir si un conocimiento puede ser patentado.

f) Necesidad de capacitación.

g) Roles de los actores del centro de estudio respecto al repositorio

Cabe agregar que durante la investigación estuvo presente el proceso de consulta de fuentes de información diversas y en variados soportes, donde se incluyó también la documentación del centro de estudio, por esta vía se obtuvo la 
información sobre el comportamiento de la producción científica del centro de estudios CAD/CAM por categorías académica y científica y por grupos de publicaciones, entre otras. A partir de otras fuentes fue posible conocer las apreciaciones de autores como Rieger (2008) que tratan los grupos sociales relevantes, asimismo fue consultado Bijker (1995) sobre la importancia de estudiar los sentidos y apreciaciones que los grupos sociales relevantes atribuyen a la tecnología porque son elementos que determinan su éxito o fracaso.

Por otra parte Hernández Pérez, Rodríguez Mateos y Bueno de la Fuente (2007) fueron utilizados para estudiar las tareas que debe emprender la biblioteca en la conformación de un RI. Asimismo se tomaron elementos aportados por la Confederación de Repositorios de Acceso Abierto COAR (2013) para el estudio de los tipos de mandatos en diferentes universidades del mundo. Fue fundamental la consulta de la Política para el desarrollo de la red de repositorios digitales institucionales del Ministerio de Educación Superior de 2015, como guía para su aplicación en la Universidad de Holguín, tomando en consideración las particularidades de esta institución. Con estos elementos fue posible interpretar y contextualizar la información para la conformación del marco teórico, la determinación de las variables a trabajar para diagnosticar el conocimiento sobre el RI de los sujetos estudiados y para diseñar e implementar el RI.

Se aplicó además la investigación - acción durante ambas etapas. Según Legrá y Silva (2010: 207) este es un método considerado ejemplar dentro de los enfoques cualitativos y sociocríticos de investigación porque tiene como propósito no sólo la comprensión, interpretación y reflexión, sino además la transformación de la realidad estudiada... no se propone obtener generalizaciones sino la comprensión y descripción exacta de los hechos estudiados para acometer su solución. Continúan los autores indicando que, en este caso, el investigador es un participante comprometido, quien al mismo tiempo que conduce la solución del problema y la transformación del objeto, se transforma a sí mismo.

Esto fue realizado en todo el decurso la investigación y como resultados de la aplicación de los diferentes métodos, técnicas e instrumentos de investigación se lograron desarrollar acciones de capacitación, que unidas al diseño, implantación y desarrollo del RI del centro de estudio transformaron la situación en la que se encontraba la producción científica, situándola en un nuevo estadio que garantiza su preservación, acceso y visibilidad, de la misma manera los bibliotecarios transformaron sus conocimientos y en gran medida su forma de hacer el trabajo. Un resumen de los métodos, técnicas e instrumentos de investigación se muestran en la tabla 1. 
Tabla 1. Métodos, técnicas e instrumentos de investigación.

\begin{tabular}{|c|c|c|c|c|c|}
\hline Etapas & Objetivos & Métodos & Técnicas & Instrumentos & $\begin{array}{c}\text { Técnicas de } \\
\text { análisis }\end{array}$ \\
\hline \multirow[b]{2}{*}{ I } & \multirow{2}{*}{$\begin{array}{l}\text { Diagnostica } \\
\text { r el } \\
\text { conocimient } \\
\text { o sobre los } \\
\text { RI de los } \\
\text { sujetos } \\
\text { investigados }\end{array}$} & \multirow{2}{*}{ Empíricos } & $\begin{array}{l}\text { Cuantitativa } \\
\mathrm{s}\end{array}$ & $\begin{array}{c}\text { Cuestionario } \\
\text { (preguntas cerradas } \\
\text { y abiertas) a los } \\
\text { profesores del } \\
\text { centro de estudio }\end{array}$ & $\begin{array}{l}\text { Categorización e } \\
\text { interpretación de } \\
\text { datos. Estadística }\end{array}$ \\
\hline & & & $\begin{array}{l}\text { Cuantitativa } \\
\mathrm{s}\end{array}$ & $\begin{array}{c}\text { Entrevista } \\
\text { estructurada (guía } \\
\text { de la entrevista) a } \\
\text { los bibliotecarios }\end{array}$ & $\begin{array}{l}\text { Categorización e } \\
\text { interpretación de } \\
\text { datos. Estadística }\end{array}$ \\
\hline \multirow[b]{2}{*}{ II } & \multirow{2}{*}{$\begin{array}{c}\text { Diseñar e } \\
\text { implementar } \\
\text { el RI }\end{array}$} & \multirow[b]{2}{*}{ Empíricos } & Cualitativas & $\begin{array}{l}\text { Grupo focal con } \\
\text { investigadores del } \\
\text { centro de estudio } \\
\text { CAD/CAM }\end{array}$ & $\begin{array}{c}\text { Categorización e } \\
\text { interpretación de } \\
\text { datos. }\end{array}$ \\
\hline & & & $\begin{array}{l}\text { Cuantitativa } \\
\mathrm{s}\end{array}$ & $\begin{array}{c}\text { Entrevista } \\
\text { estructurada (guía } \\
\text { de la entrevista) a } \\
\text { los profesores del } \\
\text { centro de estudio }\end{array}$ & $\begin{array}{c}\text { Categorización e } \\
\text { interpretación de } \\
\text { datos. } \\
\text { Estadística }\end{array}$ \\
\hline I y II & & Empíricos & Cualitativas & $\begin{array}{c}\text { Revisión } \\
\text { bibliográfica }\end{array}$ & $\begin{array}{c}\text { Interpretación y } \\
\text { contextualización } \\
\text { de la información }\end{array}$ \\
\hline I y II & & Empíricos & Cualitativas & $\begin{array}{c}\text { Observación } \\
\text { participante (guía de } \\
\text { observación) } \\
\text { Observar el } \\
\text { comportamiento de } \\
\text { los bibliotecarios } \\
\text { con respecto al } \\
\text { trabajo en la } \\
\text { plataforma Dspace } \\
\text { antes y después de } \\
\text { las acciones de } \\
\text { capacitación } \\
\end{array}$ & $\begin{array}{c}\text { Categorización e } \\
\text { interpretación de } \\
\text { datos. }\end{array}$ \\
\hline I y II & & $\begin{array}{l}\text { Empíricos } \\
\text { Investigac } \\
\text { ión- } \\
\text { acción }\end{array}$ & $\begin{array}{l}\text { Cualitativos } \\
\text { y } \\
\text { sociocríticos }\end{array}$ & $\begin{array}{l}\text { Esquema de lo que } \\
\text { es necesario } \\
\text { solucionar y las } \\
\text { acciones para } \\
\text { hacerlo. }\end{array}$ & $\begin{array}{l}\text { Comprensión, } \\
\text { interpretación y } \\
\text { reflexión de los } \\
\text { hechos de forma } \\
\text { sistemática para } \\
\text { acometer su } \\
\text { solución }\end{array}$ \\
\hline
\end{tabular}

\section{Desarrollo}

\subsection{Primera etapa. Diagnóstico del conocimiento sobre los RI de los actores involucrados}

En la conformación de la política para la implementación del RI median aspectos culturales, aptitudinales, cognitivos, tecnológicos, éticos y gerenciales que marcan 
la necesidad de contextualizar las acciones a desarrollar, es así que se definió una etapa para diagnosticar el conocimiento que poseían tanto los trabajadores del centro de estudios como los de la biblioteca universitaria referido a los RI. El instrumento aplicado en este momento de la investigación en el centro de estudio fue el cuestionario.

\subsubsection{Comportamiento de las variables en el Centro de Estudios CAD/CAM}

El comportamiento de las variables en el Centro de Estudios CAD/CAM se ilustra en el gráfico 3. Las respuestas a la pregunta uno revelan que seis investigadores $\mathbf{( 7 5 . 0 0 \% )}$ almacenaban su producción científica en una computadora del departamento y un investigador (12.5\%) afirmó que lo hacía en versión impresa en el departamento. Uno (12.5 \%) no respondió. En cuanto a la visibilidad de los resultados científicos, 7 de los investigadores $(\mathbf{8 5 , 7 \% )}$ manifestaron que no tenía suficiente visibilidad y uno consideró que sí $(\mathbf{1 4 , 3 \%})$.

La información obtenida del ítem 3 relacionado con la forma en que un estudiante de la universidad puede acceder a los resultados de las investigaciones muestra el siguiente comportamiento: cuatro investigadores $\mathbf{( 5 0 . 0 0 \% )}$ afirmaron que accediendo a la página web del departamento, tres $(37.5 \%)$ revelaron que contactando con los profesores. Uno no respondió (12.5 \%).

Las respuestas al ítem 4 sobre los contextos en que son visibles los resultados, $\mathbf{( 1 0 0 \% )}$ de los investigadores afirmó que sólo en la comunidad universitaria. Sin embargo las respuestas a los ítems anteriores dejaron claro que aún en este contexto esa visibilidad era deficiente.

El ítem cinco se enfocó a determinar el conocimiento que los investigadores poseían en relación con lo que es un RI. En este caso cinco (62.5 \%) consideraron que son colecciones que capturan y preservan la producción intelectual de una o más universidades y tres $(37.5 \%$ ) expresaron que son FTP, esto demuestra que existe poco conocimiento de lo que es un repositorio y de las bondades que brinda.

Gráfico 3. Comportamiento de las variables estudiadas en el Centro de Estudios CAD/CAM

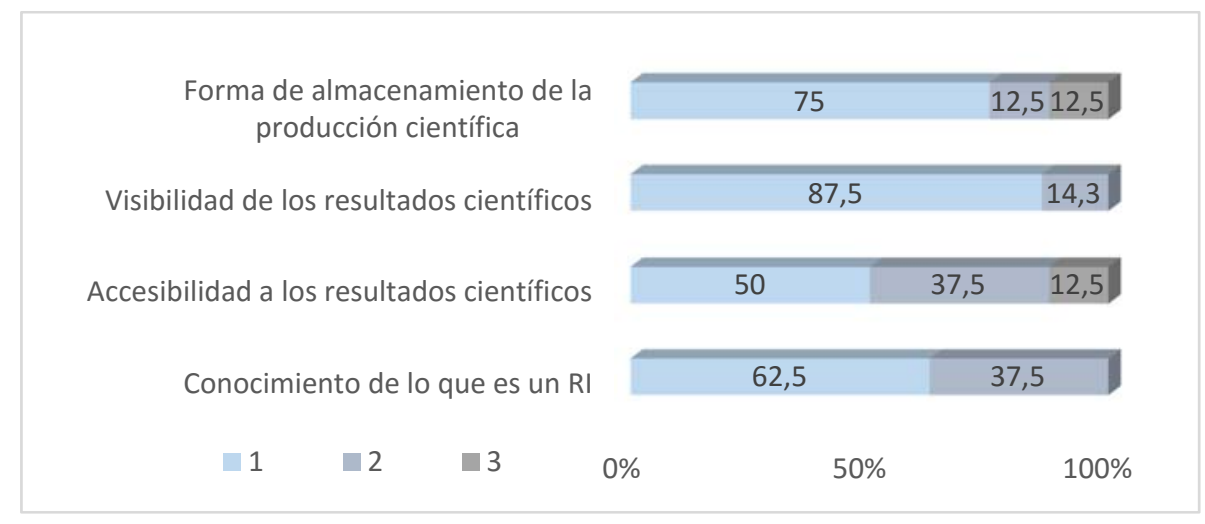


Esta situación quedó reafirmada con las respuestas al ítem seis, donde el $87.5 \%$ de los investigadores (siete) desconocían lo que es la ruta verde y dorada de acceso a la información y uno (12.5\%) si la conocía.

\subsubsection{Comportamiento de las variables en la biblioteca universitaria}

En la biblioteca universitaria se aplicó una entrevista de tipo estructurada que arrojó los resultados que se muestran en el gráfico 4 y se describen a continuación. Las respuestas al ítem uno mostró que de los quince entrevistados sólo dos $(\mathbf{1 3 , 3 3 \% )}$ respondieron de forma integral lo que es un RI, el resto $13 \mathbf{( 8 6 . 6 6 \% )}$ no lo conocían.

Respecto al ítem dos, siete trabajadores señalaron que se debían poner tesis de maestría, tesis doctorales y tesis de grado (46.66 \%), cinco no sabían (33.33\% y 3 no respondieron $\mathbf{( 2 0 . 0} \%)$. De esta forma se puede considerar que existía desconocimiento acerca de la variedad de documentos que deben subirse al RI.

La respuesta al ítem tres muestra que $\mathbf{1 0 0} \%$ de los entrevistados manifestó que no conocían la ley de derecho de autor. Mientras el $\mathbf{1 0 0 \%}$ respondió al ítem cuatro diciendo que desconocían las políticas de los repositorios.

Respecto a los roles del profesional de la información tratados en el ítem cinco, 6 trabajadores $(\mathbf{4 0 . 0} \%)$ afirmaron que no sabían cuáles son, $9(\mathbf{6 0 . 0} \%)$ dijeron que son nuevos roles pero que no los conocían y uno no respondió $(\mathbf{6 . 6 6} \%)$.

A la pregunta seis sobre las dudas o temores al enfrentar la tarea del trabajo en el repositorio, $6 \mathbf{( 4 0 . 0} \%)$ trabajadores afirmaron que su desconocimiento de lo que son los repositorios y cómo se trabaja con ellos, 2 (13.33 \%) dijeron que no estaban las condiciones tecnológicas creadas y 7 no respondieron $\mathbf{( 4 6 . 6 6 \% )}$. Respecto al ítems siete, el $\mathbf{1 0 0 \%}$ de los trabajadores afirmaron que era necesaria una capacitación.

Gráfico 4. Comportamiento de las variables estudiadas la biblioteca universitaria

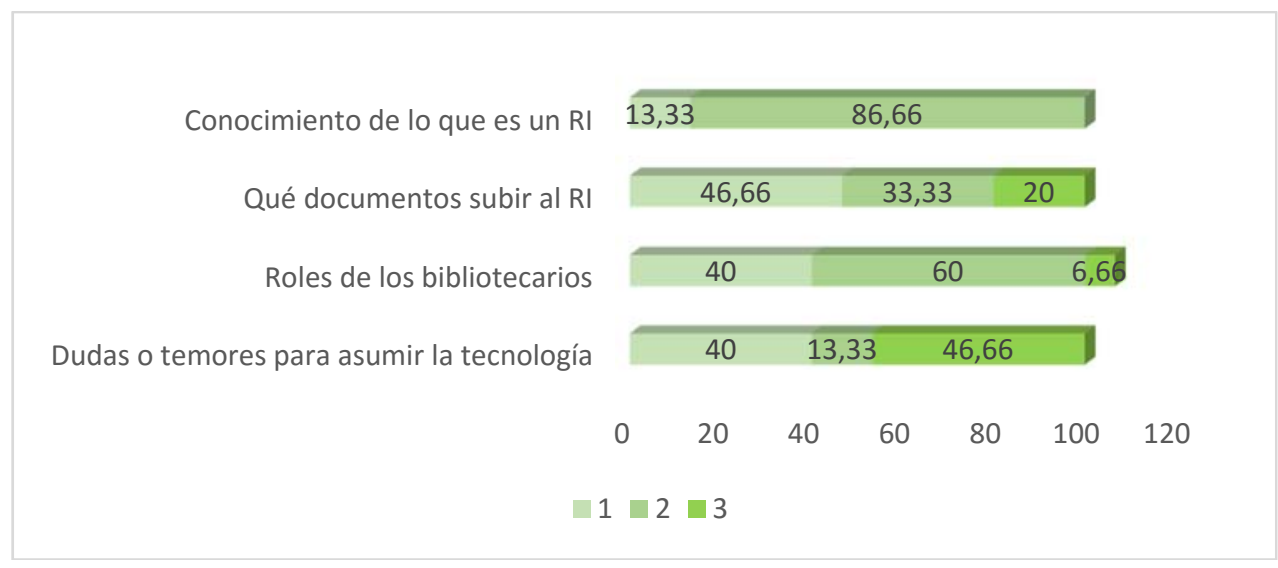


En general los resultados obtenidos revelaron la necesidad de la implementación del repositorio como vía para organizar, visualizar, preservar y propiciar el acceso a la producción científica de los investigadores y colaboradores del centro de estudios. Asimismo se demostró la necesidad de capacitar a los actores tanto del centro de estudios como de la biblioteca para que pudieran apropiarse de la tecnología desde el conocimiento de todos los beneficios y también los riesgos que puede representar el acceso abierto a la información.

Por otra parte fue evidente la necesidad de una mayor promoción de los servicios bibliotecarios y la formación de nuevas habilidades y conocimientos en los trabajadores de la biblioteca que les permitan sumarse de manera efectiva a los nuevos enfoques de la gestión universitaria del conocimiento y la innovación. Esta es una institución que puede aportar mucho en el afán de lograr la pertinencia social universitaria pero debe renovarse y alinearse con los proyectos de investigación que se llevan a cabo desde la academia para gestionar de forma efectiva la información y el conocimiento, de manera que éstos se reutilicen y desarrollen. Los repositorios institucionales constituyen una de las herramientas a utilizar en este empeño.

En este periodo de la investigación y considerando los resultados precedentes, se desarrolló el grupo focal con los investigadores del centro de estudio. Posteriormente y contando con los conocimientos adquiridos por este grupo se les aplicó una entrevista estructurada. En el gráfico 5 se muestran los resultados y a continuación se exponen los criterios recogidos a partir del análisis de las respuestas obtenidas.

Las respuestas al ítem uno revelaron que todos los investigadores estaban de acuerdo en que deben de publicarse las tesis doctorales, tesis de maestría, patentes, artículos científicos, libros de profesores y monografías, lo que coincide casi en su totalidad con lo planteado en la política de la red de universidades. Respecto al ítems dos todos los entrevistados afirmaron que debían incluirse las investigaciones de los profesores y colaboradores del centro de estudios.

Las respuestas al ítem tres revelaron que tres investigadores afirmaron que no deben ser publicados los trabajos de diplomas que obtengan 3 puntos $(\mathbf{3 7 . 5} \%)$ y cinco aseveraron que sí (62.5\%), pero que deben ser revisados por un tribunal.

Respecto al ítem cuatro el $100 \%$ de los encuestados coincidieron en que si el centro de estudios dejara de existir las colecciones deben de estar en la biblioteca de la universidad. Las respuestas a esta pregunta reafirman los vacíos de conocimiento acerca de las particularidades de los RI universitarios.

Las respuestas al ítem cinco demuestran que los investigadores saben lo que deben hacer en caso de que existan contenidos con posibilidad de ser patentados, en este caso el $\mathbf{1 0 0} \%$ responde que deben ser patentados antes de publicarse.

Las respuestas al ítems seis corroboran la necesidad de capacitación sobre aspectos como: propiedad intelectual, derecho de autor y acceso abierto a la información. El $100 \%$ de los entrevistados afirman que necesitan una capacitación para el uso del repositorio.

En cuanto a la variable roles de los actores el $75.0 \%$ (6 investigadores) afirmaron que todos deben ser responsables de subir sus trabajos al repositorio, 
mientras dos investigadores $\mathbf{( 2 5 . 0}$ \%) consideraron que deben ser responsables todos pero que el director debe controlar lo que se va a publicar.

Gráfico 5. Comportamiento de las variables estudiadas mediante la entrevista en el centro de estudios CAD/CAM.

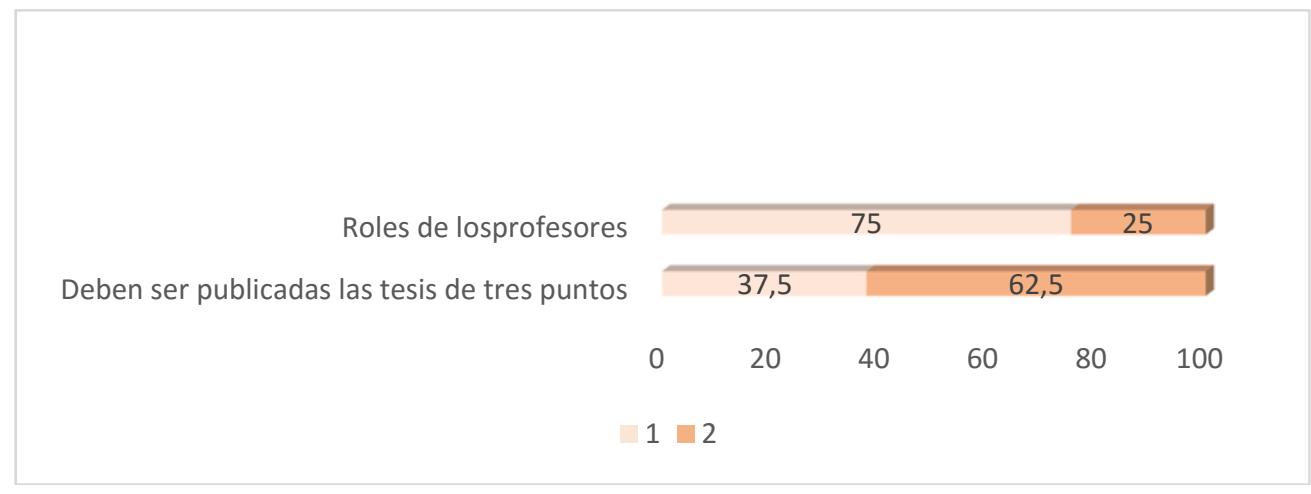

\subsection{Segunda etapa. Diseño e implementación del RI del Centro de Estudios CAD/CAM. Contextualization de la política de la Red de Repositorios Digitales de Información (RRDI)}

Tomando en consideración los resultados de la primera etapa del estudio y siguiendo la política asumida por la Red de Repositorios Digitales de Información (RRDI), se desarrolló esta segunda etapa del trabajo que concluyó con la implementación del RI del Centro de Estudios CAD/CAM.

A partir del año 2013 comenzaron las reuniones de trabajo, talleres y comunicación permanente entre los actores de las universidades cubanas y belgas; entre los principales objetivos de esta etapa estaba la conformación de la política para la red de repositorios, lo que junto al proceso de introducción de esta tecnología en las bibliotecas cubanas partícipes del proyecto se caracterizó por el carácter participativo en un escenario que propició la visión del trabajo en red y el enfoque colaborativo, lo que puede considerarse como buenas prácticas de la gestión universitaria del conocimiento. La versión definitiva de la política de la Red de Repositorios Digitales de Información (RRDI) estuvo lista totalmente en enero de 2015, sin embargo por el carácter participativo que tuvo su conformación fue posible comenzar las acciones de implementación en la Universidad de Holguín, específicamente en el Centro de Estudios CAD/CAM, desde mucho antes.

\subsubsection{Adhesión de la alta dirección de la universidad}

La política asumida por la Red de Repositorios Digitales de Información (RRDI) y su derivación a la Universidad de Holguín fue presentada a diferentes actores clave 
como el vicerrector de investigaciones y postgrados y en diferentes escenarios universitarios como el Consejo de Dirección del área de Gestión de Información Científica y en el Seminario de preparación para los cuadros, hasta que finalmente se presentó ante el Consejo de Dirección de la Universidad y posteriormente se aprobó por la Resolución rectoral No 29672016.

\subsubsection{Definición de la proyección estratégica}

En esta fase se definió la misión, visión y objetivos del repositorio con el fin de contar con los mayores detalles posibles de lo que se desea conseguir. La definición de estos debe estar en concordancia con las necesidades antes identificadas y alineada con la proyección estratégica de la universidad.

Misión: Garantiza el libre acceso a la información para satisfacer los intereses y necesidades informativas de la comunidad universitaria, local e internacional; preserva y visualiza la producción científica de los investigadores del centro de estudios CAD/CAM.

Visión: Garantiza el libre acceso a la información para satisfacer los intereses y necesidades informativas de la comunidad universitaria, local e internacional; preserva y visualiza la producción científica de los investigadores del centro de estudios, genera estadísticas que permiten medir el impacto de dichas investigaciones y caracteriza el capital humano mediante el currículo de sus investigadores lo que potencia la conformación de redes de conocimientos intra, inter y extrauniversitarias

\section{Objetivos:}

1. Socializar a nivel nacional e internacional la producción científica del centro de estudios.

2. Preservar la producción científica y académica.

3. Elevar la visibilidad nacional e internacional de la producción científica y académica.

\section{a) Estructura del repositorio}

En la figura 1 se muestra la estructura del RI del centro de estudios. Las tesis doctorales de los investigadores de este centro se incluyen en la colección Tribunal de Ingeniería Mecánica, que es el tribunal nacional, las tesis defendidas en el extranjero se incluyen en esta colección pues es este el tribunal que homologa el título. Se busca con esto evitar la dispersión de contenidos similares en varias colecciones dentro del repositorio y facilitar el acceso a la información por parte de los usuarios. La Comunidad Tesis de Maestría contiene las colecciones referidas al programa de maestría "Diseño y fabricación asistido por computadoras para la rama metalmecánica (CAD/CAM)".

En la Comunidad Otros documentos científicos del RI de la Universidad de Holguín (RI-UHO) se creó una Subcomunidad para el Centro de Estudios $\mathrm{CAD} / \mathrm{CAM}$ en la que se incluyen las siguientes colecciones: Artículos científicos, Ponencias de eventos, Informes de investigación, Monografías, Libros, Capítulos de Libros y Patentes. 
Si el centro de estudios dejara de existir se colocará la siguiente notación: (Hasta año), lo cual es indicativo de que luego de esa fecha dejó de existir esta subcomunidad.

\section{b) Definición de los roles}

La determinación del papel que cada actor involucrado debe desempeñar es fundamental para el éxito de la gestión de información y conocimientos desde el repositorio. Es así como quedó conformado el equipo de trabajo de la siguiente manera:

\section{Por el Centro de Información:}

1. Coordinador/a del Repositorio Institucional de la Universidad de Holguín: Coordina el proyecto 3 y es responsable de todas las acciones del Repositorio Institucional.

2. Coordinador/a y responsable del Repositorio del Centro de Estudios CAD/CAM: Coordina todas las acciones que se realicen con el Centro de Estudios CAD/CAM.

Realiza el archivo mediado de las colecciones preexistentes (aquellos documentos que son previos a la implementación del repositorio). Auxilia al informático en la gestión del grupo de discusión.

3. Revisor de los metadatos: Examina la normalización y calidad de los metadatos introducidos y permite la entrada de los documentos al repositorio.

4. Informático: Responsable de instalar la plataforma Dspace v4.0 y de su mantenimiento, administración y migración a otras versiones si así es considerado. Asimismo realiza copias de toda la información contenida en el repositorio. Crea grupos de discusión para dar respuesta a las dudas sobre el proceso de autoarchivo, gestión de los metadatos y otros relacionados con el repositorio.

5. Certificador de publicaciones: Responsable de emitir el certificado de publicación, después que los artículos publicados por los investigadores estén en el repositorio.

\section{Por el Centro de Estudios CAD/CAM}

1. Coordinador y responsable del RI del Centro de Estudios CAD/CAM: Responsable de entregar las colecciones de documentos preexistentes a la Biblioteca en la primera etapa de desarrollo del repositorio, cuando se aplicó el archivo mediado. 


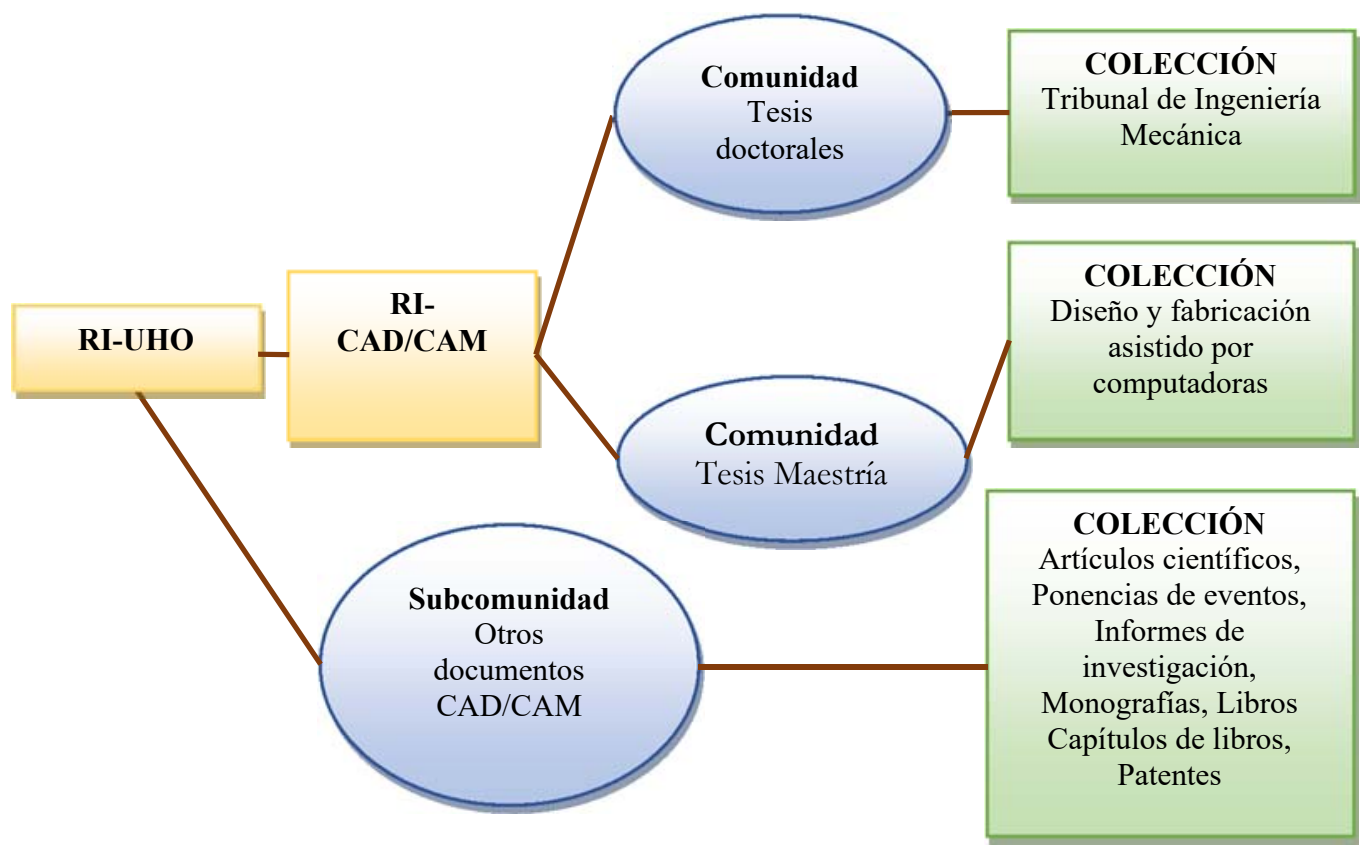

Figura 1. Estructura del Repositorio de Información del Centro de Estudios CAD/CAM

En la segunda etapa o etapa de autoarchivo es la persona responsable de que todos los investigadores y profesores del centro de estudio realicen el autoarchivo de su producción científica.

2. Investigadores y profesores: Son responsables de realizar el autoarchivo de su producción científica y de asignar los metadatos con la calidad requerida.

Asesor jurídico: Persona encargada de brindar asesoría relacionada con aspectos legales como el derecho de autor y otros aspectos que sean necesarios.

\section{c) Flujo documental y de información}

El flujo de la documentación se muestra en la figura 2. Los documentos que estaban almacenados en el centro de estudios fueron entregados por su director a la especialista responsable por la biblioteca que se encargó de realizar el archivo mediado y asignar los metadatos, éstos fueron revisados y fueron subidos al repositorio por el revisor y publicados. En el caso de no cumplir con la calidad son retenidos y se revisan nuevamente los metadatos. En la etapa de autoarchivo (todavía no implementada), los investigadores subirán su producción científica y aceptarán la licencia de depósito, posteriormente ocurre el resto del proceso tal como sucede en la etapa de archivo mediado, pero en este caso los autores deben garantizar la calidad de los metadatos. 


\section{d) Plataforma informática y disponibilidades tecnológicas}

La Red de Repositorios Digitales Institucionales ha definido que se utilice la plataforma Dspace v4.0, a partir de las siguientes disponibilidades. Es un software de código abierto diseñado por el MIT y los laboratorios de HP, se ha convertido en herramienta para la administración de colecciones digitales y por ello usado como solución para la implementación de RI. Permite gestionar repositorios de ficheros (textuales, audio, vídeo), facilitando su depósito, organizándolos en comunidades, asignándoles metadatos y permitiendo su difusión a recolectores o agregadores. Estas características han hecho que sea uno de los programas preferidos por las instituciones académicas para gestionar sus repositorios, por ser una de las más idóneas para que los investigadores depositen sus publicaciones y materiales de búsqueda con objeto de darles una mayor visibilidad.

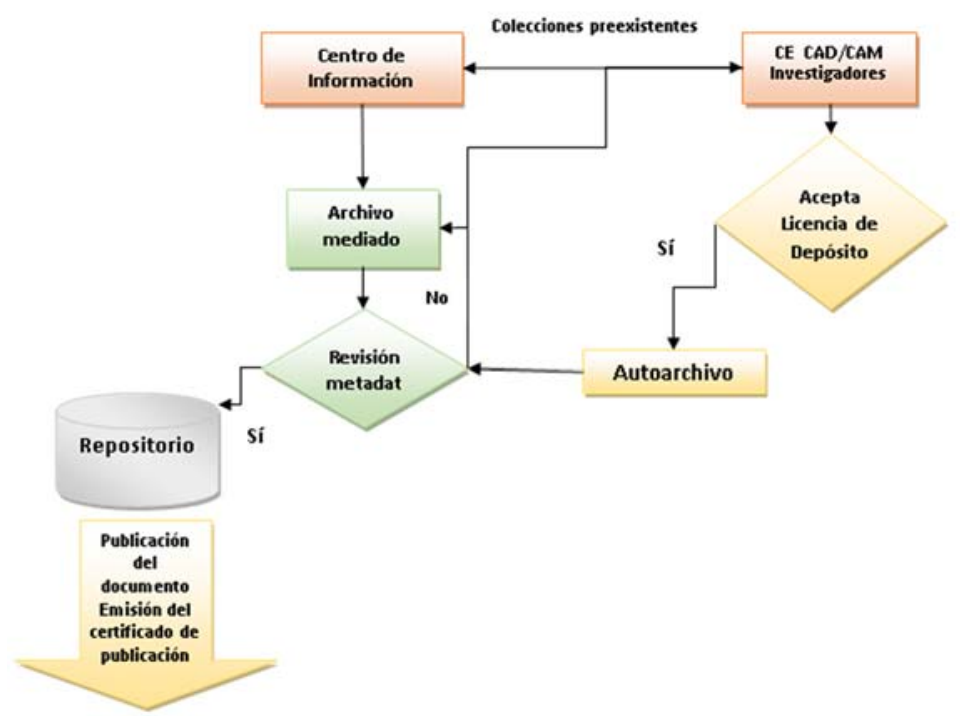

Figura 2. Flujo documental y de información del RI Centro de Estudios CAD/CAM

Además esta herramienta ofrece muchas otras funcionalidades como aquella que permite que los datos sean organizados como ítems que pertenecen a una colección, y cada colección pertenece a una comunidad; permite elegir el tipo de descripción de los recursos por tipología de fuentes de información y manejar estándares de protocolos para la interoperabilidad.

Respecto a este último aspecto, esta investigación considera que el protocolo OAI-PMH (Open Archives Initiative-Protocol Metadata Harvesting), es el indicado para garantizar la interoperabilidad que requiere un RI con otros sistemas de información y redes de repositorios nacionales y regionales. 


\section{Marketing}

Las acciones de marketing tienen como mercado meta a todos los investigadores y profesores del centro de estudios y a la alta dirección de la universidad. Este proceso comenzó desde los primeros contactos con los investigadores y directivos.

Otras acciones se diseñaron a partir de la identificación de los vacíos de conocimientos manifestados en el diagnóstico, entre ellas:

Crear grupos de discusión para el usuario desde la página Web de la biblioteca.

Crear poster informativos en las áreas de la biblioteca y otras mayormente frecuentadas.

Publicitar los nombres de las áreas y los profesores e investigadores más destacados en el autoarchivo de documentos, a través de la página Web de la biblioteca y la de la universidad, como reconocimiento a estas actitudes.

\section{e) Retroalimentación}

Se aplicará una encuesta semestralmente para conseguir información del nivel de satisfacción de los investigadores del centro de estudios respecto al desarrollo del trabajo con el repositorio y a partir de los resultados se realizarán mejoras.

Se mantienen contactos sistemáticos con el director y coordinador por el centro de estudios para verificar el cumplimiento de la política implementada y recoger propuestas de mejoras.

Se aplicará una encuesta semestralmente para conseguir información del nivel de satisfacción de los usuarios de las colecciones.

\section{f) Sostenibilidad del repositorio}

Existe un equipo de desarrollo de software, teniendo en cuenta la lista de necesidades de la RRDI que también brinda el soporte técnico.

\section{Resultados}

Resultado del desarrollo de la primera etapa. Diagnóstico del conocimiento sobre los RI de los actores involucrados, fue posible planificar y desarrollar un programa de formación para los trabajadores de la biblioteca conformado por conferencias y clases prácticas. Los temas y objetivos de las mismas se indican a continuación:

Conferencia 1: Repositorios institucionales nuevas formas de comunicación científica: ruta verde. Dspace como plataforma para los RI.

Objetivo: Explicar el papel de los RI como forma de comunicación de los resultados científicos dentro de la denominada ruta verde de acceso a la información y el papel de la plataforma Dspace para su gestión.

Conferencia 2: Nuevos roles del profesional de la información en la sociedad del conocimiento.

Objetivo: Explicar los nuevos roles del profesional de la información y mostrar las perspectivas que ofrecen las TIC como plataforma para la gestión de la información. 
Conferencia 3: Ley de derecho de autor en Cuba.

Objetivo: Explicar elementos de la ley de Derecho de autor en Cuba y su incidencia en las políticas de acceso abierto.

Se desarrollaron además una serie de clases prácticas impartidas por el informático responsable del RI.

\section{Clases prácticas}

Fueron desarrolladas tres clases prácticas para diferentes grupos de la biblioteca.

Clase práctica 1: Trabajo en la plataforma Dspace.

Objetivo: Incorporar registros de tesis de maestrías, doctorales y de pregrado al repositorio para adquirir las habilidades necesarias para el trabajo posterior en el mismo.

De esta forma los bibliotecarios quedaron listos para desarrollar la etapa de archivo mediado de las colecciones prexistentes que se encontraban en el centro de estudio. Para apoyarlos en este trabajo fue conformado el manual de procedimientos del repositorio.

A partir de la segunda etapa de la investigación "Diseño e implementación del RI del Centro de Estudios CAD/CAM. Contextualization de la política de la Red de Repositorios Digitales de Información (RRDI)", fue posible concientizar a los directivos universitarios, sumarlos y logar la probación de la política.

Asimismo quedó definida la estructura del repositorio, el flujo documental y los roles de los actores, tanto bibliotecarios como profesores. Igualmente quedaron lista tanto la plataforma como los recursos humanos para poder desarrollar el trabajo. Actualmente todas las fuentes de información que fueron entregadas a la biblioteca están disponibles en las colecciones y es posible consultarlas en la dirección http://repositorio.uho.edu.cu/jspui/

En consecuencia están implementados los servicios de búsqueda, recuperación, navegación, visualización de los contenidos, búsquedas avanzadas por comunidad, sub-comunidad, colecciones y autor y notificación de recepción de contenidos.

Actualmente se desarrollan las actividades de formación y promoción para lograr que los profesores realicen el autoarchivo de su producción científica. Por otra parte será necesario desarrollar estudios de usabilidad para conocer en qué grado el repositorio está siendo utilizado por los usuarios, qué eficacia, eficiencia y qué grado de satisfacción tienen respecto al mismo. Al mismo tiempo está en desarrollo la implantación de VIVO, como aplicación web semántica para visualizar y conectar el conocimiento dentro y fuera del campus universitario.

\section{Conclusiones}

El objetivo de la primera etapa de la investigación se cumplió al diagnosticarse el conocimiento que sobre los RI presentaban, tanto los trabajadores del centro de estudios como los de la biblioteca universitaria. En el caso del centro de estudios los resultados permitieron constatar que la producción científica se encontraba en riesgo al no ser segura su preservación, además con barreras para la visualización y uso por 
parte de otros investigadores y estudiantes y de la comunidad extrauniversitaria. Asimismo existía desconocimiento de la proyección que se lleva a cabo desde la biblioteca universitaria en cuanto al RI y sobre lo que es un RI y las posibilidades que brinda para la visualización de la producción científica. Igualmente había desconocimiento de lo que significa el movimiento de acceso abierto a la información como vía alternativa de comunicación de la ciencia y todos los sujetos investigados reconocieron la necesidad de realizar con ellos acciones de capacitación.

El estudio en la biblioteca reveló que los bibliotecarios desconocían lo que es un RI y las fuentes de información que se almacenan en los mismos. Mostraron desconocimiento de la ley de Derecho de autor en Cuba y las políticas para implementar los RI. Asimismo solo pudieron expresar que los RI significan asumir nuevos roles pero desconocían cuáles por lo que la necesidad de conocer les creaba dudas o temores para enfrentar esta tarea, así como la no existencia de toda la tecnología necesaria.

Con los resultados obtenidos fue posible planificar y desarrollar un programa de formación para los trabajadores de la biblioteca conformado por conferencias y clases prácticas que los preparó para desarrollar la etapa de archivo mediado, asimismo se obtuvieron elementos que permitieron cumplir con el objetivo de la segunda etapa.

El cumplimiento del objetivo de la segunda etapa se cumplió al quedar contextualizada la política asumida por la Red de Repositorios Digitales de Información (RRDI) a la Universidad de Holguín desde un enfoque participativo donde quedó definida la estructura del repositorio, el flujo documental y los roles de los actores, tanto bibliotecarios como profesores. De esta forma quedaron lista tanto la plataforma como los recursos humanos para poder desarrollar el trabajo. Actualmente todas las fuentes de información que fueron entregadas a la biblioteca por el director del centro de estudio están disponibles en sus colecciones y es posible su consulta en línea en la dirección http://repositorio.uho.edu.cu/jspui/ donde se puede realizar la búsqueda, recuperación y visualización de los contenidos, búsquedas avanzadas por comunidad, sub-comunidad, colecciones y autor y se realiza la notificación de recepción de contenidos.

\section{Referencias bibliográficas}

Álvarez Fernández, L.C. (2016). El acceso abierto y los repositorios digitales en las universidades cubanas. VII Congreso Internacional de Tecnologías y Contenidos Multimedia "La Habana 14-18 de marzo. <www.informaticahabana.cu/sites/default/ files/Programa\%20Cient\% C3\%ADfico\%20XVII\%20Congreso\%20Internacional\% $20 \mathrm{de} \% 20$ Tecnolog\%C3\%ADas\%20y\%20Contenidos\%20Multimedia_0.pdf.> [Consulta: 20/02/2017]

Babini D., González, J., López, F. y Medici, F. (2010). Construcción social de repositorios institucionales: el caso de un repositorio de América Latina y el Caribe. Información, Cultura y Sociedad. (23) <www.aacademica.org/fernando.ariel.lopez/3.pdf>. [Consulta: 03/10/2013] 
Bijker, W.E, Hughes, T.P, \& Pinch, T. (1995).Of bicycles, bakelites, and bulbs: Toward a theory of sociotechnical change. Cambridge, MA: MIT Press. $<$ www.prism.gatech.edu/ hk28/Klein02-SciTechHumanVal.pdf $>$

CAD/CAM (2015). Informe del Centro de Estudios CAD/CAM 2010-2014. Universidad de Holguín. [Consulta: 18/12/2014].

Castaño Muñoz, W. y Restrepo, C. (2016). Los repositorios como herramienta para la recuperación del patrimonio bibliográfico: el caso de seis bibliotecas públicas municipales del departamento de Antioquia. Revista Interamericana de Bibliotecología.

COAR (2013). Incentivos, Integración y Mediación: Prácticas sostenibles para poblar repositorios. Confederación de Repositorios de Acceso Abierto (COAR) <http://coarrepositories.org >. [Consulta: 18/12/2014]

Crow, R, (2002). The Case for Institutional Repositories: A SPARC Position Paper. Washington: Scholarly Publishing \& Academic Resources Coalition (SPARC). 37. $<$ https://ils.unc.edu/courses/2015_fall/inls700_001/Readings/Crow2002CaseforInstitutionalRepositoriesSPARCPaper.pdf $>$. [Consulta: 01/03/2014]

Hernández Pérez, T, Rodríguez Mateos, D, y Bueno de la Fuente, G. (2007). Open Access: El papel de las bibliotecas en los repositorios institucionales de acceso abierto. Anales de Documentación. <http://revistas.um.es/analesdoc/article/view/1141>. [Consulta: 03/06/2014]

Legrá, A. A. y Silva, O. (2010). La Investigación Científica: Conceptos y Reflexiones. La Habana, Editorial Félix Varela.

Lynch, A. (2003). Institutional Repositories: essential infrastructure for scholarship in the Digital Age. ARL, no. $226<$ https://www.cni.org/wp-content/uploads/2003/02/arl-br226-Lynch-IRs-2003.pdf>.

Ministerio de Justicia (2010). Gaceta oficial de la República de Cuba. Decreto Ley No $271 / 2010$ de las Bibliotecas de la República de Cuba $<$ http://legislacion.sld.cu/index.php?P=FullRecord\&ID=97>. [Consulta: 02/16/2015]

Núñez Jover y Castro Sánchez, F (2005). Universidad, Sociedad e Innovación: Experiencias de la Universidad de la Habana. Revista de Ciências da Administração, 7(13), $\quad 1-21<$ https://periodicos.ufsc.br/index.php/adm/article/view/2325/2032>. [Consulta: 03/06/2015]

Rieger, O. Y. (2008). Opening Up Institutional Repositories: Social Construction of Innovation in Scholarly Communication. The Journal of Electronic Publishing 11(3).

Thomas, H. (2009). De las tecnologías apropiadas a las tecnologías sociales. Conceptos / estrategias / diseños / acciones. Grupo de Estudios Sociales de la Tecnología y la Innovación IEC/UNQ, CONICET.

Universidad Central de Las Villas (2015). Política para el desarrollo de la red de repositorios digitales institucionales del Ministerio de Educación Superior. 